\title{
PREPARATION OF MAGNETO-OPTICAL COATED FILMS WITH Bi-YIG PARTICLES
}

\author{
E. KOMURO, T. HIRANO*, N. KAWAI, T. NAMIKAWA and Y. YAMAZAKI \\ Tokyo Institute of Technology, Nagatsuta, Midori-ku, Yokohama 227, Japan \\ *Toppan Printing Co., Ltd., Takanodai-minami, Sugito, Saitama 345, Japan
}

\begin{abstract}
Garnet fine particles are prepared by coprecipitation and annealing. The saturation magnetization of the particles increases as the annealing temperature increases. Almost all X-ray diffraction peaks from the particles annealed at $700{ }^{\circ} \mathrm{C}$ for $1 \mathrm{~h}$ are assigned to garnet. Faraday rotation of the particles which are dispersed into methylene iodide is measured under a polarization microscope. The Faraday rotation of the coated films are shown as a function of wavelength. Existence of small fine particles of about $50 \mathrm{~nm}$ diameter is confirmed in the coated film by SEM observation. A magnetic field pattern was displayed using the coated film prepared.
\end{abstract}

KEYWORDS : Bi-YIG, COPRECIPITATION, FARADAY ROTAION, COATED THIN FILM, DISPLAY DEVICE, PARTICLES, MAGNETO-OPTICAL PROPERTIES

\section{INTRODUCTION}

Thin films of Bi substituted yttrium iron garnet (Bi-YIG) are applicable to magneto-optical disks and magneto-optical display devices. In the preparation of Bi-YIG thin films by sputtering methods, ceramic crystal or glass substrates are required because of the high preparation temperature. As the temperature is normally higher than $500{ }^{\circ} \mathrm{C}$, it is difficult to use the plastic substrates. Recently garnet fine particles were obtained by chemical methods [1], [2]. It is possible to avoid the thermal requirement by adopting the coating method in which Bi-YIG fine particles are dispersed in binders [3]. Therefore, garnet fine particle; are considered to be a promising material for thin film applications [3], [4].

We prepare Bi-YIG fine particles by a coprecipitation method, and discuss their structural and magnetic properties. Faraday rotation of the film containing the particles is measured in the visible wavelength region.

\section{EXPERIMENTAL}

Figure 1 shows the preparation process of $\mathrm{Bi}-$ YIG particles by coprecipitation [5]. First, aqueous solutions of nitrates of $\mathrm{Bi}, \mathrm{Y}$ and $\mathrm{Fe}$ with the ratio of cations corresponding to the composition of $\mathrm{Bi}_{1.6} \mathrm{Y}_{1.4} \mathrm{Fe}_{5} \mathrm{O}_{12}$ were prepared and mixed at room temperature with an alkaline solution of $\mathrm{NH}_{4} \mathrm{OH}$. After the coprecipitation reaction, changing the $\mathrm{pH}$ value of the solution alters the composition and the saturation magnetization of the particles. The dependence of the composition and the saturation magnetization of the particles on the $\mathrm{pH}$ of the solution is indicated in Fig. 2. In the range of the $\mathrm{pH}$ over 8.7, the ratio of the cations in the particles was the same as that of the nitrate solution. In this study, the $\mathrm{pH}$ of the solution was fixed to 10.7. Then the obtained slurry was washed with water to remove the alkaline ions, filtered and dried at $100{ }^{\circ} \mathrm{C}$ for $1.5 \mathrm{~h}$. Then the coprecipitate was annealed in air at $\mathrm{Ta}=500{ }^{\circ} \mathrm{C} \sim$ $700{ }^{\circ} \mathrm{C}$ for $1 \mathrm{~h}$ or $4 \mathrm{~h}$ to crystallize (Ta: annealing temperature).

The composition of the coprecipitates was analyzed by inductively coupled plasma spectroscopy. The shape and the size of the particles were investigated using a transmission electron microscope

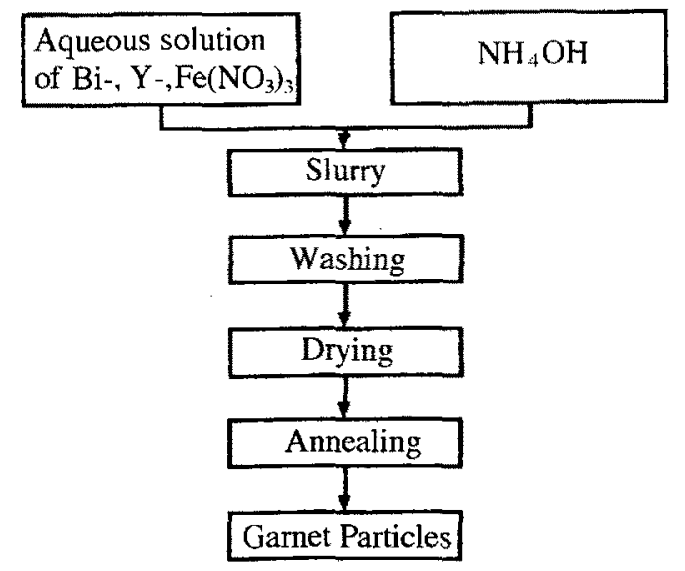

Fig. 1 Preparation of garnet fine particles by coprecipitation. 
(TEM) and the crystal structure of the particles was examined by $\mathrm{X}$-ray diffraction. Their magnetic properties were investigated using a vibrating sample magnetometer at room temperature.

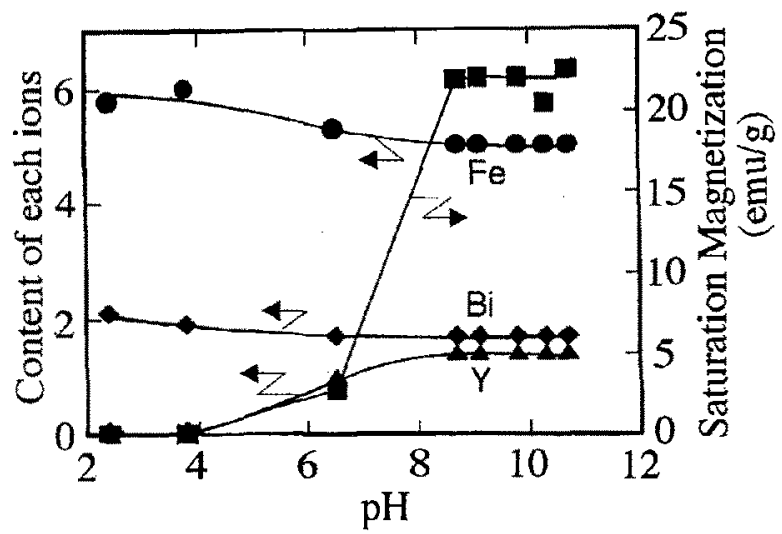

Fig. 2 Content of each ions and saturation magnetization of the particles as a function of $\mathrm{pH}$ of the solution.

The coated magneto-optical thin films were prepared as follows. First, the particles, organic binder and cyclohexanone were mixed. Then it was milled for $10 \mathrm{~h}$ and coated on a glass substrate using a spin coater. The film thickness was adjusted by the speed of rotation of the spin coater. A homogeneous film was obtained.

\section{RESULTS AND DISCUSSION}

\section{Magnetic Properties}

Figure 3 shows the saturation magnetization of the particles as a function of the annealing temperature for different two annealing periods. For annealing period of $1 \mathrm{~h}$, magnetization appears at $\mathrm{Ta}=580{ }^{\circ} \mathrm{C}$,

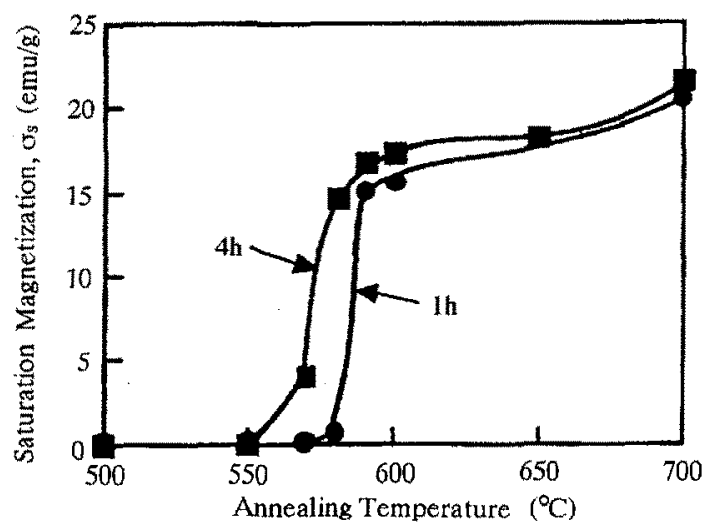

Fig. 3 Saturation magnetization of preparation particles vs. annealing temperature for different annealing periods. whereas for $4 \mathrm{~h}$, the magnetization appears at $\mathrm{Ta}=570$ ${ }^{\circ} \mathrm{C}$.

X-ray diffraction patterns of the particles annealed for $4 \mathrm{~h}$ at various temperatures from $500{ }^{\circ} \mathrm{C}$ to $700{ }^{\circ} \mathrm{C}$ are shown in Fig. 4 . At $550{ }^{\circ} \mathrm{C}$, no peaks were detected. Garnet peaks appear at $600{ }^{\circ} \mathrm{C}$, but some unidentified peaks are also present. Then, at $700{ }^{\circ} \mathrm{C}$, the unidentified peaks become negligibly small and almost all peaks are assigned to garnet. The particles annealed for $1 \mathrm{~h}$ show the same tendency as those annealed for $4 \mathrm{~h}$ in regard to $\mathrm{X}$-ray diffraction.

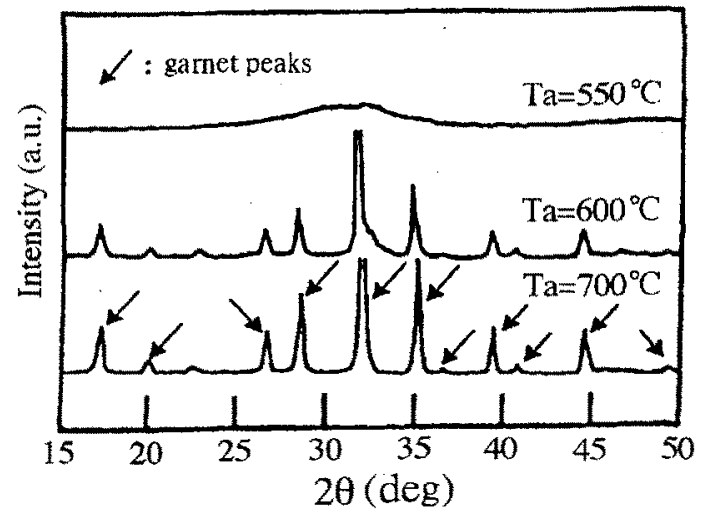

Fig. $4 X$-ray diffraction patterns of the particles for various annealing temperature.

Figure 5 shows TEM micrographs of the particles annealed for $4 \mathrm{~h}$ at various temperatures from $500{ }^{\circ} \mathrm{C}$ to $700{ }^{\circ} \mathrm{C}$. The magnetization is not detected in the particles annealed at $500{ }^{\circ} \mathrm{C}$ and $550{ }^{\circ} \mathrm{C}$.

Some particles, in the powder samples synthesized at $570{ }^{\circ} \mathrm{C}$ for $1 \mathrm{~h}$, are found to show considerably large magneto-optical effect.

\section{Observation of the particles}

Images of the particles annealed at $700{ }^{\circ} \mathrm{C}$ for 1h dispersed in methylene iodide are shown in Fig. 6 . The photos were taken using a polarization micro-

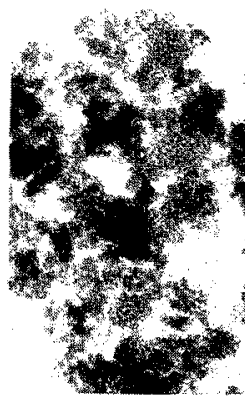

$\mathrm{Ta}=550^{\circ} \mathrm{C}$

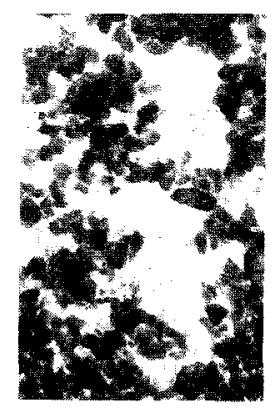

$\mathrm{Ta}=600^{\circ} \mathrm{C}$

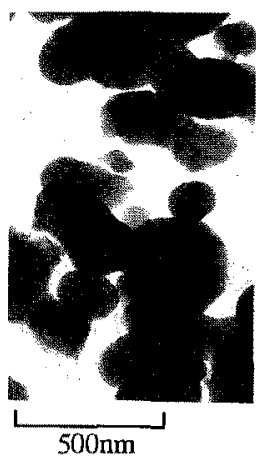

$\mathrm{Ta}=700^{\circ} \mathrm{C}$
Fig. 5 TEM micrographs of annealed Bi-YIG particles. 


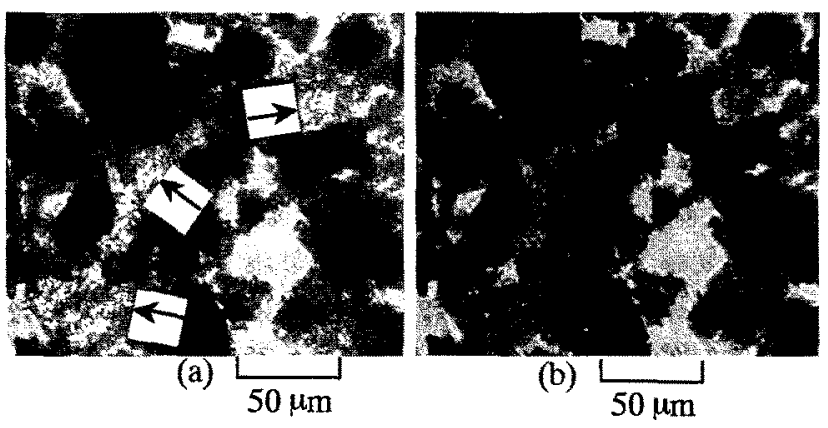

Fig. 6 Images obtained with annealed Bi-YIG particles, immersed in methylene iodide under obverse (a) and reverse (b) magnetic fields.

scope, under opposite magnetic fields. The analyzer was fixed at five degrees from crossed Nicol condition. Methylene iodide was used as a dispersion medium because of its high refractive index. The particles which the arrows point to in Fig. 6a are bright while those in Fig. $6 \mathrm{~b}$ are dark. This result suggests that some of the synthesized particles rotate the plane of polarization of visible wavelength light.

\section{Faraday rotation of coated film}

Figure 7 shows the Faraday rotation of the film containing the particles having the composition of $\mathrm{Bi}_{1.8} \mathrm{Y}_{1.2} \mathrm{Fe}_{5} \mathrm{O}_{12}$ at $1000 \mathrm{rpm}$ as a function of wavelength. The thickness of the film was about $630 \mathrm{~nm}$. In the region of the wavelength from $420 \mathrm{~nm}$ to $570 \mathrm{~nm}$, the coated film showed Faraday rotation.

\section{Optical Read Out of Magnetic Pattern}

The scanning electron microscope (SEM) image of the film coated at $100 \mathrm{rpm}$ is shown in Fig. 8 . Thickness of the film is $1.5 \mu \mathrm{m}$. Small fine particles which are about $50 \mathrm{~nm}$ diameter are observed. It is

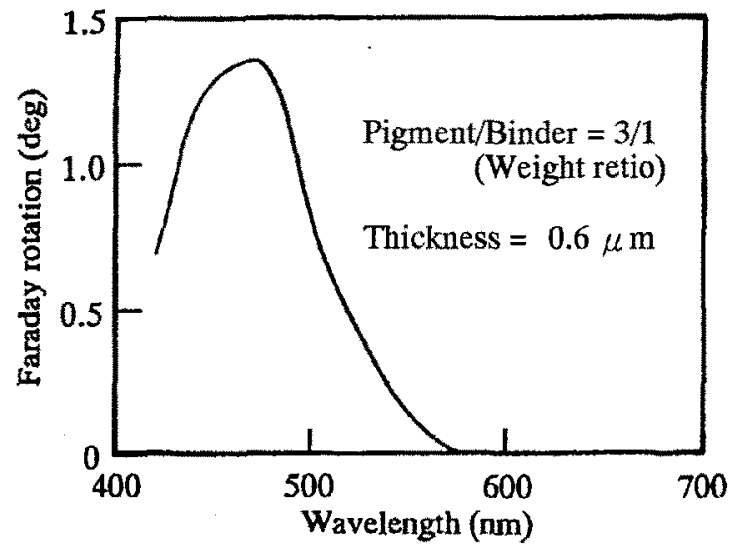

Fig. 7 Faraday rotation of the film prepared by spin coating at $1000 \mathrm{rpm}$ as a function of wavelength. confirmed that the particles are uniformly dispersed.

The Bi-YIG containing film was mounted on a mirror which was placed on a magnet. The magnet was magnetized with a checkered pattern as shown in Fig. 9. The optical arrangement is also shown in Fig. 9. The angles of the analyzer were \pm 2 degrees off-set from crossed Nicol condition for the read out experiment.

Figure 10 shows the read out patterns obtained with the reflected light. The magnetic pattern of the magnet placed under the film was detected using the prepared film. It is also noted that the read out is carried out with an optic arrangement of reflection configuration which is more suitable for optical devices than that of transmission configuration.

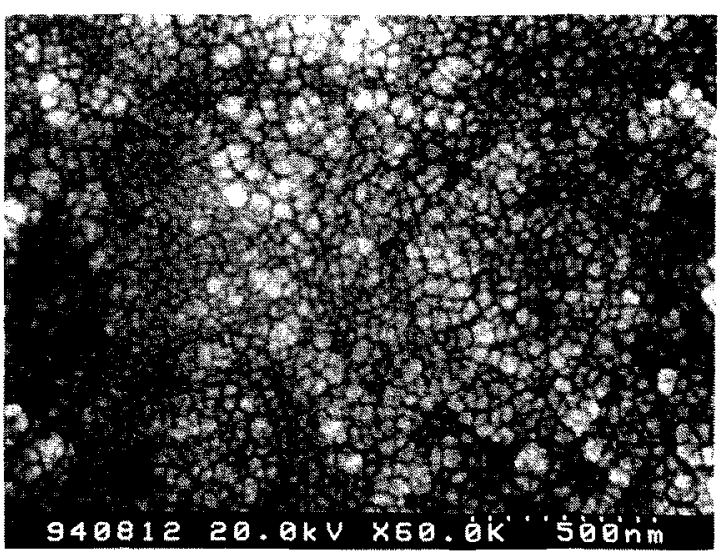

Fig. 8 SEM image of the film coated at $100 \mathrm{rpm}$ with spin coater.

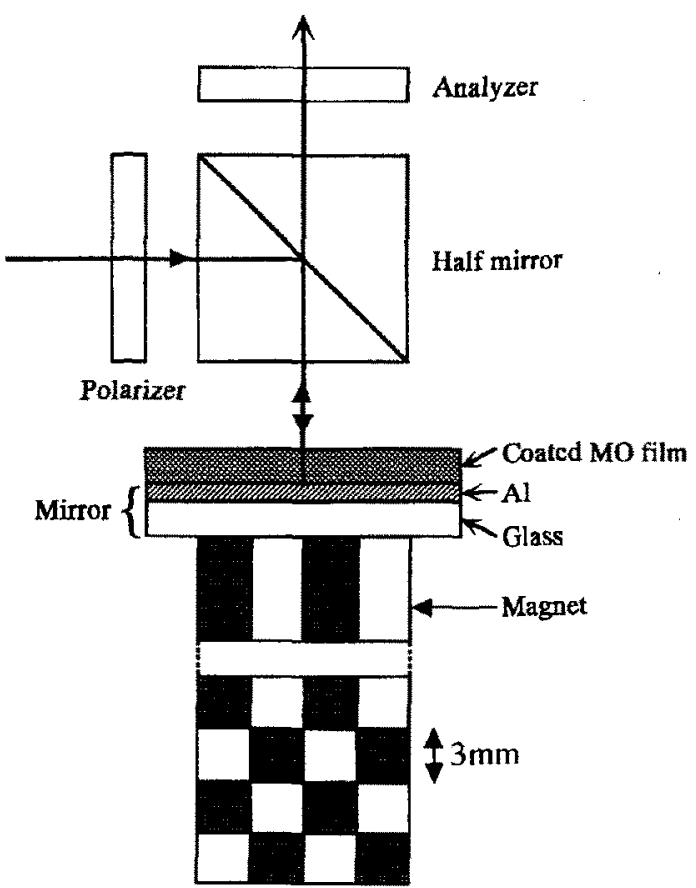

Fig.9 Optical system for the read out of magnetic pattern. 


\section{CONCLUSIONS}
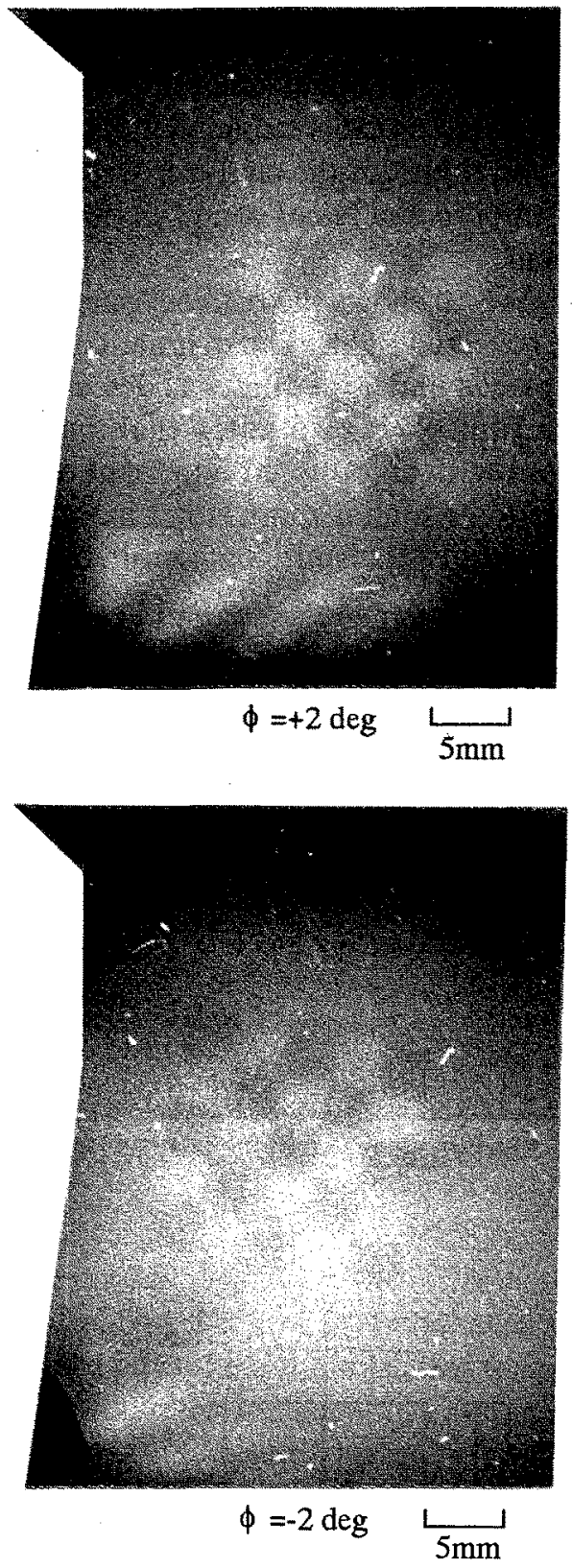

Fig. $10 \mathrm{Bi}-$ YIG coated films showing a magnetic field pattern for reflected light.
Bi-YIG fine particles were prepared by coprecipitation and anneal process in order to study their structural, magnetic and magneto-optical properties. From the results of measurements for saturation magnetization and X-ray diffraction patterns of the particles, it was found that garnet phase appears over $550^{\circ} \mathrm{C}$, and pure garnet phase is formed at $700{ }^{\circ} \mathrm{C}$. Faraday rotation of the particles was detected using a polarization microscope. A MO film was prepared by dispersing the particles in a binder. The film showed Faraday rotation in the region of wavelength from $420 \mathrm{~nm}$ to $570 \mathrm{~nm}$. From SEM analysis, it is confirmed that the film is homogeneous and consists of small fine particles of about $50 \mathrm{~nm}$ diameter. The coated MO film showed magnetic field pattern for reflected light. Thus, Bi-YIG fine particles prepared by coprecipitation process have the possibility to be applied to display devices working in the visible wavelength region.

\section{REFERENCES}

[1] M. Gomi, Serada and M. Abe, "Fine particles of Bi- and Ce- substituted iron garnet prepared by coprecipitation," Proceedings of The Sixth International conference on Ferrites, 999 (1992).

[2] K. Odagawa, K. Nakagawa and A. Itoh, "Effects of heat treatment conditions and impurity doping on garnet films for M-O recording prepared by pyrolysis," IEEE Trans. Magn., vol. 26, 1721 (1990).

[3] T. Fujimoto, Y. Kumura, M. Gomi and M. Abe, "Particulate films for magneto-optical recording III and IV," J. Magn. Soc. Jpn., vol. 15, Sppl. S1, 263 and 267 (1991).

[4] K. Nakagawa and A. Itoh, "Microstructure and crystallization mechanism of Bi-substituted garnet films for $\mathrm{M}-\mathrm{O}$ recording prepared by pyrolysis and sputtering," J. Magn. Soc. Jpn., vol. 17, Sppl. S1, 278 (1993).

[5] N. Kawai, T. Hirano, E. Komuro, T. Namikawa and Y. Yamazaki, "Preparation of Bi substituted YIG particles by coprecipitation," DENKI KAGAKU, vol. 62, 348 (1994). 